\title{
Arrhythmias and Sudden Death among Older Children and Young Adults Following Tetralogy of Fallot Repair in the Current Era: Are Previously Reported Risk Factors Still Applicable?
}

\author{
Swati Arya, MD, Julie Kovach, MD, Harinder Singh, MD, and Peter P. Karpawich, MSc, MD \\ Section of Pediatric Cardiology, Carmen and Ann Adams Department of Pediatrics, The Children's Hospital of \\ Michigan, Wayne State University School of Medicine, Detroit, Mich, USA
}

\section{A B S T R A C T}

Background. Young adult patients (pts) with repaired tetralogy of Fallot (TOF) remain at risk for arrhythmias (Ar) and sudden cardiac death (SCD). Based on past studies with earlier pt subsets, Ar/SCD events were associated with right ventricular (RV) systolic pressures $>60 \mathrm{~mm} \mathrm{Hg}$, outflow tract gradients $>20 \mathrm{~mm} \mathrm{Hg}$, and QRS duration $>180 \mathrm{~ms}$. However, there are limited recent studies to evaluate these risk factors in the current patient generation. Methods. Patients with TOF followed over the past 50 years were grouped by presence of any arrhythmias (group 1), absence of arrhythmias (group 2), and presence of SCD or significant ventricular arrhythmias (group 3) and correlated with current pt age, gender, age at repair, repair types, echocardiogram, cardiac magnetic resonance imaging, electrocardiogram/Holter, hemodynamics, and electrophysiology findings.

Results. Of 109 pts, 52 were male aged 17-58 years. Of these, 59 (54\%) had Ar, two of whom had SCD. These 59 pts were chronologically older at the time of analysis, with repair at an older age and wider QRS duration (78-240, mean $158 \mathrm{~ms}$ ) when compared with those without Ar. However, there was no correlation with surgical era, surgical repair, gender, $\mathrm{RV}$ pressure $>60 \mathrm{~mm} \mathrm{Hg}$, right ventricular outflow tract gradient $>20 \mathrm{~mm} \mathrm{Hg}$, or RV end-diastolic volume on CMRI.

Conclusions. Ar/SCD risk continues to correlate with repair age and advancing pt age. QRS duration is longer in these patients but at a shorter interval (mean $158 \mathrm{~ms}$ ) and less RV pressure (mean $43 \mathrm{~mm} \mathrm{Hg}$ ) than previously reported. In the current TOF patient generation, neither surgical era, type of repair, RV outflow gradient nor RV volume correlate with Ar/SCD. Electrophysiologic testing to verify and identify arrhythmias remains clinically effective.

Key Words. Tetralogy of Fallot; Adult Congenital Heart; Sudden Death; Arrhythmias

\section{Introduction}

urgical repair of tetralogy of Fallot (TOF), the $\checkmark$ most common cyanotic congenital heart disease, has seen tremendous advances since first performed 50 years ago and is now performed earlier than in previous decades. Successful surgery has led to an increasing population of adults with repaired TOF. However, with this increased life expectancy, this group of patients (pts) also suffers from late complications, notably arrhythmias resulting in morbidity and mortality often years after repair. ${ }^{1,2}$ Studies in the 1980 s reported an increased risk of arrhythmias and sudden cardiac death (SCD) associated with certain clinical risk factors: late definitive repair

(C) 2013 Wiley Periodicals, Inc. following an initial palliation, presence of a transannular patch, significant pulmonary and/or tricuspid insufficiency, severe right ventricular (RV) and right atrial enlargement, elevated right ventricular systolic pressure (RVSP), increased right ventricular outflow tract (RVOT) pressure gradient, and a prolonged QRS duration. ${ }^{3-8}$ Electrophysiological studies (EPSs) have been shown to have prognostic value in identifying pts at risk for significant arrhythmias. ${ }^{9}$ More recently, focus has shifted toward discerning the role of left ventricular dysfunction in arrhythmia generation. ${ }^{10}$ However, despite the availability of several techniques, no single variable has been identified to be predictive of arrhythmias potentially predisposing to SCD among patients with repaired TOF. The

Congenit Heart Dis. 2014;9:407-414 
purpose of this study was to reevaluate the previously reported risk factors and to determine if those still hold true in the current era.

\section{Materials and Methods}

A retrospective chart review was conducted on all pts above 17 years of age with at least 10 years of postsurgical follow-up with repaired TOF/ pulmonary atresia (PA) followed at the Michigan Adult Congenital Heart Clinic of the Children's Hospital of Michigan, Detroit Medical Center. The study was approved by the Wayne State University Institutional Review Board. Patients who did not have complete repair and had only a palliative shunt as well as those followed elsewhere postoperatively, and in whom clinical data was not available, were excluded. A total of 109 pts meeting the 10 -year postrepair inclusion criteria were identified, all of whom had undergone surgical repair between 1970 and 1995 and were followed at the Children's Hospital of Michigan Section of Cardiology with available clinical data. This accounted for approximately three fourth of all patients who had undergone complete surgical TOF repair during that time interval and subsequently followed at our institution. Reviewed clinical data included patient complaints/symptoms including fatigue, palpitations, sustained arrhythmias, syncope/presyncope, and any evidence of aborted sudden death. Noninvasive testing (electrocardiogram [ECG], ambulatory monitoring/ Holter and exercise stress testing) and Doppler/ echocardiographic data (chamber dimensions, contractility indices, RVSPs, RVOT pressure gradients, valve integrity, and left ventricular function) were reviewed. Data obtained during cardiac catheterization included all hemodynamic measurements, and results of EPSs were recorded. Patients were grouped by presence of atrial and/or ventricular arrhythmias (group 1), absence of arrhythmias (group 2), and presence of SCD or significant ventricular arrhythmias (group 3), which was a subgroup of group 1.

\section{Statistical Analysis}

Data were analyzed using SPSS software (version 20; SPSS Inc, Chicago, IL, USA). Fisher's exact test was used to compare categorical data, and two-tailed Student's $t$-test was used to compare means. Results are presented as mean value \pm standard deviation. Significance was defined as a $P$ value $<.05$. The $95 \%$ confidence interval was calculated wherever appropriate.

\section{Results}

\section{Demographics}

A total of 109 pts were identified, all of whom underwent surgical repair between 1970 and 1995 . The mean age of the cohort was $29 \pm 11$ years (median age: 27 years, range 17-58) of whom 52 $(48 \%)$ were male. Mean age at intracardiac repair was $41.2 \pm 48$ months (median: 24, range 1-216 months). Mean years of follow-up postintracardiac repair was $23.4 \pm 8$ years (median: 22, range 10-50 years). Uncomplicated TOF was seen in 93 pts (85\%), whereas 16 pts (15\%) had pulmonary atresia with VSD (PA-VSD). An initial aortopulmonary shunt prior to intracardiac repair was performed in 54 pts $(50 \%)$ : 50 of those were Blalock-Taussig-Thomas (BTT) shunts (classic and modified) and four were central aortopulmonary shunts (Waterston or Potts). Outflow tract repair consisted of a transannular patch in 67 pts $(61 \%)$, whereas 11 pts $(10 \%)$ were repaired without a transannular patch. A right ventricle to pulmonary artery conduit was performed in 27 (25\%) pts: $11(10 \%)$ of these patients with TOF and $16(15 \%)$ with PA-VSD (Table 1$)$.

\section{Patients and Complaints}

Of the 109 patients, 73 (67\%) were clinically symptomatic with complaints of fatigue, palpitations, syncope, or presyncope. Of these complaints, fatigue only was the most common, occurring in 50 (46\%). At the time of their latest evaluation, 59 patients were in functional New York Heart Association (NYHA) class I, 44 were in NYHA class II, three were in NYHA class III, and three were in NYHA class IV. The median age of patients in NYHA class I (26, range 17-58 years) did not differ significantly with patients in NYHA class II (29, range 17-51 years). The small number of patients with NYHA III and IV precluded any effective comparisons of median ages with other groups. Other complaints including palpitations were seen among 32 (29\%) patients. Bonafide arrhythmias that included supraventricular tachycardia in the form of atrial flutter or intra-atrial reentrant tachycardia, ventricular ectopy defined by Lown's criteria class C (multifocal premature ventricular complexes [PVCs], couplets/triplets), nonsustained or sustained ventricular tachycardias or ventricular fibrillation, and/or aborted SCD were documented in 59 (54\%) patients (group 1). In spite of their subjective complaints, including sensed tachycardias, 50 patients (group 2) exhibited no arrhythmias by clinical testing. 
Table 1. Comparative Demographic, Clinical, Echocardiographic, Electrocardiographic, and MRI Data among Patients with/without Arrhythmia

\begin{tabular}{|c|c|c|c|}
\hline & Group 1 (Arrhythmias) & Group 2 (No Arrhythmias) & $P$ value \\
\hline \multicolumn{4}{|l|}{ Demographic data } \\
\hline Number of patients & 59 & 50 & \\
\hline Mean age (years) & $32.9(11.1)$ & $25(8.3)$ & .0001 \\
\hline Mean follow-up (years) & $25.7(8.9)$ & $20.6(7.1)$ & .003 \\
\hline \multicolumn{4}{|l|}{ Surgical characteristics } \\
\hline Mean age at repair (months) & $50.4(53.3)$ & $29.5(38.2)$ & .03 \\
\hline Prior aortopulmonary shunt & $30(59 \%)$ & $24(51 \%)$ & 1.0 \\
\hline \multicolumn{4}{|l|}{ Type of repair } \\
\hline Transannular patch & $34(61 \%)$ & $33(67 \%)$ & .5 \\
\hline No transannular patch & $06(11 \%)$ & $05(10 \%)$ & 1.0 \\
\hline RV to PA conduit & $16(29 \%)$ & $11(22 \%)$ & 1.0 \\
\hline \multicolumn{4}{|l|}{ Echocardiography } \\
\hline Patients with RVSP $\geq 60$ & $7(17 \%)$ & $5(14 \%)$ & .7 \\
\hline Mean RVSP (mm Hg) & $42.5(16.5)$ & $37.1(13.2)$ & .1 \\
\hline Patients with RVOT gradient $\geq 20$ & $12(32 \%)$ & $9(24 \%)$ & .4 \\
\hline Mean RVOT gradient (mm Hg) & $16.8(12.1)$ & $17.6(16.8)$ & .8 \\
\hline Patients with moderate to severe PI & $34(68 \%)$ & 35 (74\%) & .5 \\
\hline Patients with moderate to severe RV dilatation & $26(47 \%)$ & $26(55 \%)$ & .4 \\
\hline Patients with moderate to severe RA dilatation & $11(20 \%)$ & $11(23 \%)$ & .8 \\
\hline Mean LVEF (\%) & $61.9(7.9)$ & $64.3(7.7)$ & .2 \\
\hline Mean FS (\%) & $34.8(5.0)$ & $34.9(5.8)$ & .9 \\
\hline \multicolumn{4}{|l|}{ Electrocardiogram } \\
\hline Patients with QRS duration $\geq 180 \mathrm{~ms}$ & $14(27 \%)$ & $3(7 \%)$ & .01 \\
\hline Mean QRS duration (ms) & $158(28)$ & $149(19)$ & .07 \\
\hline \multicolumn{4}{|l|}{ MRI } \\
\hline RVEDVi $\left(\mathrm{mL} / \mathrm{m}^{2}\right)$ & 162 & 154 & .6 \\
\hline RVEF (\%) & 38.1 & 45 & .07 \\
\hline PRF (\%) & 53 & 44 & .08 \\
\hline
\end{tabular}

FS, fractional shortening; LVEF, left ventricular ejection fraction; PI, pulmonary insufficiency; RA, right atrium; RVOT, right ventricular outflow tract; RVSP, right ventricular systolic pressure.

There was a significant difference between groups in mean age at latest evaluation: group 1, $32.9 \pm 11$ years (median: 31 , range $17-58$ years) vs. group 2, $25 \pm 8$ (median: 22, range 17-53 years) $(P=.0001)$. Similarly, there was a significant difference between groups in regard to age at intracardiac repair $(50 \pm 53$ vs. $30 \pm 38$ months $[P=$ $.03])$. Median age at repair was also determined. Median age at repair of group 1 was 36 months (range 1-216 months) and that for group 2 was 17.5 months (range 3-204 months). Mean years of follow-up postintracardiac repair was also significantly different between the two groups (group 1: $26 \pm 9$ vs. group $2: 21 \pm 7$ years, $P=.003$ ). In group $1,30 / 51(59 \%)$ pts had an initial aortopulmonary shunt, either BTT (28) or Waterston (2) prior to intracardiac repair, whereas in group 2, 24/47 (51\%) pts underwent a shunt procedure, BTT (22) and Waterston (2) $(P=\mathrm{NS})$. Intracardiac repair consisted of a transannular patch in 34 pts from group 1 and 33 pts in group $2(P=\mathrm{NS})$. Similarly, no significant differences were found for patients without a transannular patch or right ventricle to pulmonary artery conduit repair between the two groups.

\section{SCD/Aborted SCD}

There was one sudden death and one aborted sudden death in our cohort among group 1 patients. The one patient who suffered SCD was 17 years old. He had typical TOF with a shunt surgery at 2 months of age and intracardiac repair with a transannular patch performed at 18 months of age. He presented in refractory ventricular fibrillation. The patient with aborted SCD was a 53-year-old male with typical TOF, who underwent an initial shunt procedure and subsequent intracardiac repair with a transannular patch. He had undergone an apparently successful ablation for ventricular tachycardia with a negative repeat EPS 4 years previously. This patient presented with ventricular tachycardia requiring cardioversion and received an implantable cardiac defibrillator (ICD).

\section{Ultrasound and Catheterization Data}

Based on echocardiographic end-diastolic RV dimensions, 52 patients had evidence of moderate to severe RV dilation, 26 (47\%) of whom were in group 1 and $26(55 \%)$ in group 2. This difference was not significant $(P=.4)$. Accordingly, moderate to severe right atrial dilation was 
seen in 22 of the 109 patients with, again, no significant differences between groups (11 vs. 11, $P=.8)$. Moderate to severe tricuspid insufficiency was seen in nine patients (group $1=4$; group $2=$ $5, P=.7)$. Moderate to severe pulmonary insufficiency was seen in $34(68 \%)$ of patients in group 1 and $35(74 \%)$ of patients in group $2(P=$ NS). Similarly, there was no difference in the number of patients with an estimated elevated RVSP $>60 \mathrm{~mm} \mathrm{Hg}$ (group 1: seven [17\%] and group 2: five [14\%] patients). The mean RVSP did not significantly differ between the two groups $(43.0 \pm 16 \mathrm{~mm} \mathrm{Hg}$ vs. $37.2 \pm 13 \mathrm{~mm} \mathrm{Hg}$, $P=.1)$. Similarly, for RVOT pressure gradients, there was no difference in the number of patients with RVOT gradient $>20 \mathrm{~mm} \mathrm{Hg}$ in the two groups (group 1: 12, 32\% patients and group 2: $9,24 \%$ patients). The mean RVOT gradient in group 1 was $16.8 \pm 12 \mathrm{~mm} \mathrm{Hg}$ vs. group 2: 17.6 $\pm 17 \mathrm{~mm} \mathrm{Hg}$. This difference was also statistically nonsignificant.

\section{Noninvasive Arrhythmia Evaluations}

Nearly all patients exhibited a typical postsurgical right bundle branch block ECG pattern. QRS durations were measured directly from their latest electrocardiogram. Among patients in group 1, 14/51 (27\%) had a QRS duration $\geq 180 \mathrm{~ms}$, whereas only $3 / 43(7 \%)$ in group 2 exhibited that finding. This difference was found to be statistically significant $(P=.01)$. However, the mean QRS duration was not found to be significantly longer $(P=.07)$ in group $1(158 \pm$ $28 \mathrm{~ms})$ compared with group $2(149 \pm 19 \mathrm{~ms})$. Evidence of actual arrhythmias was obtained from combined ECG, stress tests, and ambulatory monitoring. These findings included multifocal PVCs, couplets, triplets, ventricular tachycardia and fibrillation, atrial reentry tachycardia, atrioventricular (AV) node reentry tachycardia, and atrial flutter and fibrillation. Of the 59 patients with documented arrhythmias, 38 (64\%) exhibited a ventricular substrate, 14 (24\%) atrial, five (9\%) both atrial and ventricular, and the remaining two patients had only evidence of AV node dysfunction (second or higher degree of AV block). Among the patients with a ventricular substrate, 11 patients were in Lown's grade C, class 3 (multifocal PVCs), 15 patients were in grade $\mathrm{C}$, class $4 \mathrm{a}$ and $4 \mathrm{~b}$ (couplets/triplets), and 15 patients had evidence of nonsustained/ sustained ventricular tachycardia or ventricular fibrillation.

\section{Electrophysiology Data}

Intracardiac EPS were performed in 35/59 (59\%) patients with evidence of clinical arrhythmias, primarily to discern the possibility of ablation and/or need for an ICD placement. Referral for intracardiac study was at the discretion of the primary cardiologist. Comparable clinical arrhythmias were induced in 27 (77\%) of these patients. Inducible atrial arrhythmias occurred in 10 patients: intraatrial reentrant tachycardia (six), $\mathrm{AV}$ nodal reentry (one), atrial fibrillation (one), His bundle/ junctional (one), and ectopic atrial tachycardia (one). Sustained ventricular arrhythmias were induced in 17 patients; 16 of these arrhythmias were monomorphic, whereas one was polymorphic degenerating to ventricular fibrillation. Among these ventricular arrhythmias, 11 were entrained from the RVOT and/or VSD patch margin, two from RV apex, and four from the left ventricle.

$\mathrm{AV}$ node dysfunction was documented in three patients although clinical AV nodal dysfunction, as noted above, was found in eight patients. Sinus node dysfunction was found in two patients. The above numbers are not mutually exclusive. Five patients in this group with a positive EP study had a pacemaker implantation and five had ICD placements. The other 12 patients with inducible ventricular arrhythmias either had a nonsustained ventricular arrhythmia that was successfully ablated or were controlled on medications (class I, III, or beta-blockers). A negative EP study was documented in eight patients. In all patients who underwent an EP study, 5/7 (72\%) with evidence of clinical atrial arrhythmia had a positive EP study for comparable atrial arrhythmias. Similarly, for clinical ventricular arrhythmias, 17/26 (65\%) patients had a positive EP study.

\section{MRI Data}

As MRI use among patients with repaired TOF is a relatively recent clinical tool, in only a subset of our patients $(n=41)$ was data obtained. There were no significant differences in the right ventricular end diastolic volumes (RVEDVi) per patient body surface area between the two groups $\left(162 \mathrm{~mL} / \mathrm{m}^{2}\right.$ in group 1 vs. $154 \mathrm{~mL} / \mathrm{m}^{2}$ in group 2, $P=.6)$. Similarly, there were no differences in the RV ejection fraction or the pulmonary regurgitation fraction (defined as the retrograde pulmonary flow/antegrade pulmonary flow $\times 100 \%$ ) between the two groups (Table 1). 
Table 2. Comparative Demographic, Clinical, Echocardiographic, Electrocardiographic, and MRI Data among Patients with/without Ventricular Arrhythmias

\begin{tabular}{|c|c|c|c|}
\hline & Group 3 (V. Arrhythmias) & Group 2 (No Arrhythmias) & $P$ value \\
\hline \multicolumn{4}{|l|}{ Demographic data } \\
\hline Number of patients & 43 & 50 & \\
\hline Mean age (years) & $32.8(10.6)$ & $25(8.3)$ & .002 \\
\hline Mean follow-up (years) & $26.1(8.9)$ & $20.6(7.1)$ & .003 \\
\hline \multicolumn{4}{|l|}{ Surgical characteristics } \\
\hline Mean age at repair (months) & $49.9(50.7)$ & 29.5 (38.2) & .04 \\
\hline Palliation & $30(59 \%)$ & $24(51 \%)$ & 1.0 \\
\hline \multicolumn{4}{|l|}{ Type of repair } \\
\hline Transannular patch & $26(65 \%)$ & $33(67 \%)$ & .8 \\
\hline No transannular patch & $03(8 \%)$ & $05(10 \%)$ & 1.0 \\
\hline RV to PA conduit & $11(28 \%)$ & $11(22 \%)$ & 1.0 \\
\hline Prior aorto-pulmonary shunt & $23(62 \%)$ & $27(57 \%)$ & .8 \\
\hline \multicolumn{4}{|l|}{ Echocardiography } \\
\hline Patients with RVSP $\geq 60$ & $3(10 \%)$ & $5(14 \%)$ & 1.0 \\
\hline Mean RVSP (mm Hg) & $41.2(14.2)$ & $37.1(13.2)$ & .2 \\
\hline Patients with RVOT gradient $\geq 20$ & $8(32 \%)$ & $9(24 \%)$ & .5 \\
\hline Mean RVOT gradient $(\mathrm{mm} \mathrm{Hg})$ & $17.4(13.1)$ & $17.6(16.8)$ & .9 \\
\hline Patients with moderate to severe $\mathrm{PI}$ & $30(77 \%)$ & 35 (74\%) & 1.0 \\
\hline Mean LVEF (\%) & $64.1(7.5)$ & $64.3(7.7)$ & .9 \\
\hline Mean FS (\%) & $35.9(4.7)$ & $34.9(5.8)$ & .5 \\
\hline \multicolumn{4}{|l|}{ Electrocardiogram } \\
\hline Patients with $Q R S$ duration $\geq 180 \mathrm{~ms}$ & $7(19 \%)$ & $3(7 \%)$ & .1 \\
\hline Mean QRS duration (ms) & $152(25)$ & $149(19)$ & .5 \\
\hline \multicolumn{4}{|l|}{ MRI } \\
\hline RVEDVi $\left(\mathrm{mL} / \mathrm{m}^{2}\right)$ & 163 & 154 & .5 \\
\hline
\end{tabular}

FS, fractional shortening; LVEF, left ventricular ejection fraction; PI, pulmonary insufficiency; RA, right atrium; RVOT, right ventricular outflow tract; RVSP, right ventricular systolic pressure; $\mathrm{V}$, ventricular.

\section{Subgroup Analysis of Patients with Ventricular Arrhythmias}

Patients with ventricular arrhythmias (group 3) were compared with patients without arrhythmias. No difference was found between these two groups with regards to the RVSP, RVOT gradient, QRS duration, presence of moderate to severe RV enlargement, and presence of free pulmonary insufficiency (PI) and RVEDV on MRI (Table 2).

\section{Discussion}

Patients with TOF remain at high risk for morbidity and late mortality, even years after repair. ${ }^{7,11}$ Based on our current study, approximately $70 \%$ of patients present with symptoms such as fatigue, palpitations, syncope, and overt congestive heart failure. Studies in the past have shown a $10-80 \%$ incidence of significant arrhythmias in patients after TOF repair. ${ }^{2,7,12}$ Our study shows a 54\% incidence of any arrhythmias. As ventricular dysfunction and elevated pressures can contribute to atrial arrhythmias leading to patient complaints, all clinical arrhythmias were included in this study. Also, atrial arrhythmias are a source of significant morbidity in this patient population, accounting for symptoms such as dizziness and presyncope and the need for frequent cardioversion. Previ- ously, age at intracardiac repair has been shown to be an important determining factor responsible for arrhythmias in these patients. ${ }^{7,12}$ Staged repair with an initial aortopulmonary shunt has been shown to increase the risk of late arrhythmias. Chowdhury et al. in their report suggested that long-term hypoxemia resulting from the aortopulmonary shunt contributes to myocardial dysfunction and the propensity for ventricular arrhythmias. ${ }^{13}$ Repair with a transannular patch has also been implicated to increase the risk for late arrhythmias and sudden death.,14 Our study, however, does not confirm these findings. Although there was a significant difference in the age of intracardiac repair, there were no differences in history of prior aortopulmonary shunt surgery or the type of intracardiac repair between the arrhythmia and arrhythmia-free groups in our study, similar to the results reported by Harrison et al. ${ }^{15}$ Similarly, no significant difference in the incidence of arrhythmias was seen among patients who had a transannular patch during repair. Institutional operative technical differences may account for some of these differences among reported studies. Hemodynamic abnormalities in the form of an elevated RVSP $(>60 \mathrm{~mm} \mathrm{Hg})$ and an increased RVOT pressure gradient are believed to be important contributors toward arrhythmias 
and sudden death in this population. ${ }^{4,5,16}$ Our study did not show a difference in the mean RVSP between the two groups, and interestingly, the mean RVSP in both groups was much lower than the $60 \mathrm{~mm} \mathrm{Hg}$ level that has been proposed in older studies, as a risk factor for arrhythmias. Moreover, clearly, there was no correlation of arrhythmias with mean RVOT gradient, similar to the results of the study by Gatzoulis et al. ${ }^{7}$ Pulmonary insufficiency has also been implicated as an important risk factor. ${ }^{4,5,7}$ However, there was no significant difference in the number of patients with moderate to severe pulmonary insufficiency between the two groups in our study, comparable with other earlier reports. ${ }^{15,17} \mathrm{We}$ also could not establish a correlation between moderate to severe $\mathrm{RV}$ dilatation and risk of arrhythmias in general, among our study population, as shown by previous studies. ${ }^{18}$ We also studied the QRS duration of our population. Other studies have proposed the electromechanical interaction of significant pulmonary insufficiency and RV dilation contribute to deleterious EP sequelae. ${ }^{19}$ Earlier studies had shown that a QRS duration of $>180 \mathrm{msec}$ has a $100 \%$ sensitivity for sustained ventricular tachycardia and a $100 \%$ negative predictive value if it was $<180 \mathrm{msec}^{6}{ }^{6}$ A subsequent study by Gatzoulis et al., however, found that the absolute QRS duration at any given time may not be of prognostic significance. ${ }^{7}$ At their latest evaluation, three (7\%) of our patients in group 2 had a QRS duration of $\geq 180 \mathrm{msec}$, whereas $27 \%$ of the patients in group 1 had a QRS duration of $\geq 180 \mathrm{msec}$. These numbers were statistically significant. Also, the mean QRS duration was higher in the arrhythmia group (158 msec vs. $149 \mathrm{msec}$ ) although not statistically significant unlike previous studies. ${ }^{6-8,18}$ Of note, the mean QRS duration of our patients with arrhythmias is lower than that seen in previous studies as compared with Gatzoulis et al. (mean QRS duration for all arrhythmias ranges between 167 and $192 \mathrm{msec}){ }^{7}$ Recently, studies have focused on determining if left ventricular dysfunction is an important contributor toward arrhythmia generation and sudden death in this population. ${ }^{10,20}$ This report found no differences between the two groups in mean LV ejection fraction. Recognizing the fact that the risk factors for atrial and ventricular arrhythmias are different, we performed subanalysis of patients with ventricular arrhythmias with the patients with no arrhythmias and found no difference between the two groups in all the above-tested parameters. Small sample size precluded analysis of patients with only atrial arrhyth- mias and their comparison with the ventricular arrhythmia group. The importance of EP studies in predicting risk of arrhythmias and sudden death in adults with repaired TOF has been reported in some previous studies. ${ }^{9}$ However, other reports have shown that EP studies by themselves may not be sufficient to predict risk of life-threatening arrhythmias and sudden death. ${ }^{21}$ As ventricular dysfunction and elevated end-diastolic pressures can contribute to atrial arrhythmias leading to patient complaints, all clinical arrhythmias were included in this study. Also, atrial arrhythmias are a source of significant morbidity in this patient population, accounting for symptoms such as dizziness and presyncope and the need for frequent cardioversion. ${ }^{22}$ Hence, we considered it prudent to include atrial arrythmias in our arrhythmia group (group 1).

Lastly, we found a low incidence of SCD in our patient population in deference to studies from previous eras. ${ }^{1,2}$ This finding, in the current era, supports the previous suggestion that long-term relative tissue hypoxia resulting from the aortopulmonary shunt contributes to eventual myocardial cellular dysfunction and the propensity for ventricular arrhythmias that increases the risk of SCD. ${ }^{13}$

\section{Conclusion}

Results from this study reiterate the fact that patients with repaired TOF continue to be at risk from late morbidities including arrhythmias, both atrial as well as ventricular. However, a high rate of mortality was not seen in our study cohort. This study did not reproduce the previously cited correlations performed in the 1980s between type of repair and risk of arrhythmias and sudden death in this patient population. However, it should be noted that the mean age of repair in our study population was younger than that seen in those previous studies. The theory that a shorter duration of hypoxemic damage to the myocardium, as seen following a shunt procedure prior to definitive repair, appears to have some credibility. Our study did not find any correlation between the hemodynamic abnormalities and arrhythmia generation in spite of the presence of a transannular patch and free PI in a significant number of our patients. In this current study, only a longer QRS duration was convincingly found to be predictive of arrhythmias, albeit at a much lower value than previously cited in various studies. Hence, categorization of these patients for need of pulmonary 
valve replacement or other interventions based on risk for life-threatening arrhythmias could not be established. In this regard, the adult with repaired TOF remains a demanding patient group that requires continuous follow-up care.

\section{Limitations}

We encountered inherent limitations of a retrospective study from a single institution. A small sample size with limited number of adverse events was our biggest limitation. Our study group comprised a referral based biased group of patients. This would imply that asymptomatic adult patients, not currently being seen clinically, may alter the current findings if added to the patients' database. Nevertheless, the fact that all patients seen had their surgical repairs performed from a single surgical team removes some degree of technical bias to the findings.

\section{Authors' Contributions}

Swati Arya, MD: data collection, statistical analysis, and drafting the document.

Julie Kovach, MD: design and critical review.

Harinder Singh, MD: design and critical review.

Peter P. Karpawich, MSc, MD: concept, design, review, and final approval.

Corresponding Author: Peter P. Karpawich, MSc, MD, Section of Cardiology, The Children's Hospital of Michigan, 3901 Beaubien Blvd, Detroit, MI 48201, USA. Tel: (+1) 313-745-5481; Fax: (+1) 313-993-0894; E-mail: pkarpawi@dmc.org

Conflict of interest: None.

Accepted in final form: November 1, 2013.

\section{References}

1 Garson A, McNamara DG. Sudden death in a pediatric cardiology population: relationship to prior arrhythmias. $\mathcal{f}$ Am Coll Cardiol. 1988;5:134B$137 \mathrm{~B}$.

2 Deanfield JE, McKenna WJ, Presbitero P, England D, Graham GR, Hallidie-Smith K. Ventricular arrhythmia in unrepaired and repaired tetralogy of Fallot. Relation to age, timing of repair and hemodynamic status. Br Heart 7. 1984;52:77-81.

3 Garson A, Gilette PC, Gutgesell HP, McNamara DG. Stress induced ventricular arrhythmia after repair of Tetralogy of Fallot. Am 7 Cardiol. 1980;46:1006-1012.
4 Garson A, Randall DC, Gilette PC, et al. Prevention of sudden death after repair of Tetralogy of Fallot, treatment of ventricular arrhythmias. $7 \mathrm{Am}$ Coll Cardiol. 1985;6:221-227.

5 Chen D, Moller JH. Comparison of late clinical status between patients with different hemodynamic findings after repair of tetralogy of Fallot. Am Heart 7. 1987;113:757-772.

6 Gatzoulis M, Till J, Somerville J, Redington A. Mechanoelectrical interaction in tetralogy of Fallot: QRS prolongation relates to right ventricular size and predicts malignant ventricular arrhythmias and sudden death. Circulation. 1995;92:231-237.

7 Gatzoulis MA, Balaji S, Webber SA, et al. Risk factors for arrhythmia and sudden cardiac death late after repair of tetralogy of Fallot: a multicentre study. Lancet. 2000;356:975-981.

8 Tzemos N, Harris L, Carasso S, et al. Adverse left ventricular mechanics in adults with repaired tetralogy of Fallot. Am 7 Cardiol. 2008;103:420-425.

9 Khairy P, Landzberg MJ, Gatzoulis MA, et al. Value of programmed ventricular stimulation after tetralogy of Fallot repair-a multicenter study. Circulation. 2004;109:1994-2000.

10 Ghai A, Silversides C, Harris L, Webb GD, Siu SC, Therrien J. Left ventricular dysfunction is a risk factor for sudden cardiac death in adults late after repair of tetralogy of Fallot. $7 \mathrm{Am}$ Coll Cardiol. 2002;40:1675-1680.

11 Silka MJ, Hardy BG, Menache VD, Morris CD. A population based prospective evaluation of risk of sudden death after operation for common congenital heart defects. 7 Am Coll Cardiol. 1998;32:245251.

12 Nakazawa M, Shinohara T, Sasaki A, et al. Arrhythmias late after repair of Tetralogy of Fallot-a Japanese Multicenter study. Circulation. 2004;68: 126-130.

13 Chowdhury UK, Sathia S, Ray R, Singh R, Pradeep KK, Venugopal P. Histopathology of the right ventricular outflow tract and its relationship to clinical outcomes and arrhythmias in patients with tetralogy of Fallot. 7 Thorac Cardiovasc Surg. 2006;132:270277.

14 Zhao H, Miller C, Reitz B, Shumway N. Surgical repair of tetralogy of Fallot. Long term follow up with particular emphasis on late death and reoperation. 7 Thorac Cardiovasc Surg. 1985;89:204-220.

15 Harrison DA, Harris L, Siu S, et al. Sustained ventricular tachycardia in adult patients late after repair of tetralogy of Fallot. 7 Am Coll Cardiol. 1997;30: 1368-1373.

16 Kugler JD. Predicting sudden death in patients who have undergone tetralogy of Fallot repair: is it really as simple as measuring ECG intervals? 7 Cardiovasc Electrophysiol. 1998;9:103-106.

17 Zahka KG, Horneffer PJ, Rowe SA, et al. Long term valvular function after total repair of tetralogy 
of Fallot: relation to ventricular arrhythmias. Circulation. 1988;78(5 Pt 2):III14-9.

18 Daliento L, Rizzoli G, Menti L, et al. Accuracy of electrocardiographic and echocardiographic indices in predicting life threatening ventricular arrhythmias in patients operated for tetralogy of Fallot. Heart. 1999;81:650-655.

19 Dean JW, Lab MJ. Arrhythmia in hear failure: role of mechanically induced changes in electrophysiology. Lancet. 1989;i:1309-1312.

20 Broberg CS, Aboulhosn J, Gurvitz M, et al. Arrhythmia prevalance in tetralogy of Fallot relates to left sided cardiac dysfunction rather than right sided. HFSA 2010.

21 Chandar JS, Wolff GS, Garson A, et al. Ventricular arrhythmias in postoperative tetralogy of Fallot. $\mathrm{Am}$ 7 Cardiol. 1990;65:655-661.

22 Roos-Hesselink J, Perlroth MG, McGhie J, Spitaels S. Atrial arrhythmias in adults after repair of tetralogy of Fallot: correlations with clinical, exercise and echocardiographic findings. Circulation. 1995; 91:2214-2219. 